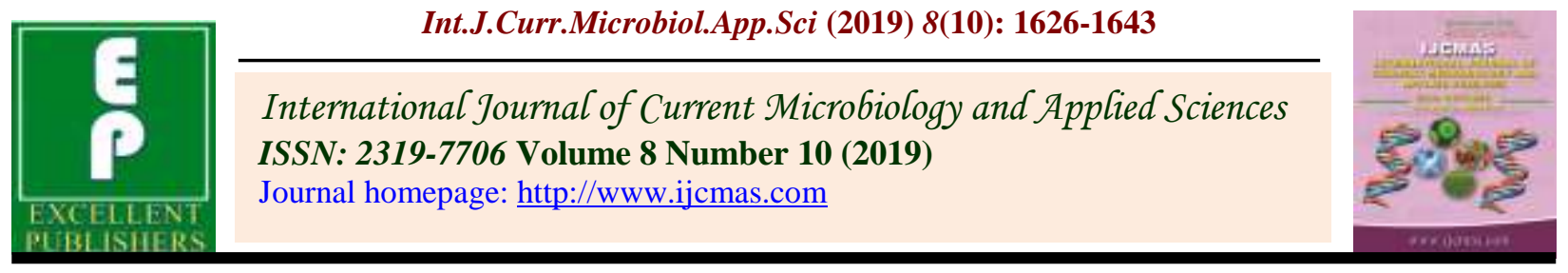

Original Research Article

https://doi.org/10.20546/ijcmas.2019.810.190

\title{
Effect of Soybean [Glycine max (L.) Merrill] based Cropping Systems on Weed Dynamics and Productivity of Soybean and Subsequent Crops of the System
}

\author{
G. S. Gathiye* and H. S. Kushwaha \\ Department of Agronomy, Mahatma Gandhi Chitrakoot Gramodaya Vishwa Vidyalaya, \\ Chitrakoot, Satna (M.P.), India \\ *Corresponding author
}

\begin{tabular}{|l|}
\hline Ke y w o r d s \\
$\begin{array}{l}\text { Soybean-based } \\
\text { cropping systems, } \\
\text { Weed dynamics, } \\
\text { Soybean equivalent } \\
\text { yield, Production } \\
\text { efficiency }\end{array}$ \\
\hline Article Info \\
\hline $\begin{array}{l}\text { Accepted: } \\
\text { 12 September } 2019 \\
\text { Available Online: } \\
\text { 10 October } 2019\end{array}$ \\
\hline
\end{tabular}

A field experiment was carried out during kharif, rabi and zaid seasons of 2015-16 and 2016-17 to study the effect of soybean [Glycine max (L.) Merrill] based cropping systems on weed dynamics and productivity of soybean and subsequent crops of the system at the research farm of Krishi Vigyan Kendra, Dhar, M.P. Among the all 16 soybean based cropping systems under Kharif, in soybean Echinochloa crusgalli was the most dominating weed contributing $27.35 \%$ of total weed intensity at most critical period (25 DAS) while Celosia argentea topped at harvest stage $(28.9 \%)$. During rabi, the relative density of weeds varied between different crops. Chenopodium album was found to be more serious weed almost in all rabi crops grown under different cropping systems. In rabi crops viz., wheat, chick pea, garlic, onion, potato and garden pea, the relative density of Chenopodium album was the most dominating 26.5, 26.75, 26.05, 28.35 $\%$, respectively at 25 DAS but the relative density of Chenopodium album slightly changed as $24.6,24.55,21.05$ and $21.70 \%$, respectively at maturity stage. During zaid season Onion and Garlic crops were grown. Cyprus rotundus contributed to $24.05 \%$ of the total weed intensity at 25 DAS in both crops, but the values of relative changed as $26.60 \%$ at maturity stage. Both varieties of soybean (JS 95-60 and JS 93-05) recorded weed intensity ranged from 224.2 to $244.5 / \mathrm{m}^{2}$. During rabi season, the weed population was significantly minimum in Soybean (JS 95-60)Potato (Kufri jyoti) - Onion (AFLR) i.e. $119.65 / \mathrm{m}^{2}$ and during zaid season, Onion significantly allowed maximum infestation of weeds $\left(120.5\right.$ to $\left.121.35 / \mathrm{m}^{2}\right)$. While comparing the total weed-intensity $/ \mathrm{m}^{2}$ for entire crop- cycle of different cropsequences, Soybean (JS 93-05) - Garlic (G-282) system had significantly minimum weed-intensification $\left(356.40 / \mathrm{m}^{2}\right)$. The weed biomass was ranged from 15.30 to $29.90 \mathrm{q} /$ ha with soybean variety JS 95-60 and JS 93-05. Among all rabi crops, the weed biomass was maximum (9.45 q/ha) under Soybean (JS 95-60) - Onion (AFLR) cropping system whereas the crop sequences consisted with potato led to record significantly lesser weed biomass $(5.35 \mathrm{q} / \mathrm{ha})$. While considering the weed biomass of entire cropping system, Soybean (JS 93-05) - Potato (Kufri jyoti) - Onion (AFLR) significantly registered the highest value $(28.90 \mathrm{q} / \mathrm{ha})$. 


\section{Introduction}

Large area under soybean is spread over Central India. Generally, it is grown as a monsoon season crop under rainfed situation mainly under Vertisols and associated soils. It has resulted increased cropping intensity and profitability. In Malwa and Nimar valley region, its cultivation is largely practiced in rainy season followed by Gram/wheat on conserved soil moisture. Under irrigated conditions, soybean is largely grown in soybean-wheat cropping system, while soybean-chickpea cropping system is prevalent under rainfed conditions. The major cropping system in the Vertisols and associated soils of Central India under regime is soybean-wheat in which soybean is a rainfed crop. Both soybean and wheat are most productive crops and predominantly grown in a sequential cropping, particularly under irrigated production system in almost all districts of Malwa plateau agro-climatic zone of Madhya Pradesh. Soybean-chickpea system is also prevalent as a next important cropping sequence mainly in those areas, where rainfall is not adequate or irrigation water is scarce. Generally, cultivation of both Soybean and wheat in a sequence are nutrients exhaustive and these crops require heavy investment in desirable agricultural operations during their cultivation. Long term regular practice of Soybean-Chickpea and Soybean-Wheat system in the growing region is posing severe problems before the growers such as complexity in weed management, deterioration of soil-properties, delayed sowing of wheat and low market value of produce owing low productivity as well as poor economic viability of this cropping system. Under such circumstances, the diversification of existing soybean wheat/chickpea system needs to be evaluated to meet the domestic need of farmers. Simultaneously, the economic status of the farmers of Soybean-wheat growing areas will also be raised by replacing any of the two crop components with the introduction of high value crop without degrading the landresources. Consequent upon above facts, evaluation of suitable diversified cropping system under existing agro-ecological and farming situation needs to be identified through proper investigation.

Weed dynamics is severely affected by cropping system and establishment techniques. Continues cultivation of same crop year after year the weed population will be same. Crop rotations affect seed banks because weed control measures change with successive crops. Weed flora have changed over the past century, with either increasing or decreasing species abundance depending on the management. Rabi crops like wheat, potato, garlic and onion crops require large quantity of irrigation water which favours build up of weed infestation in such areas. Therefore, it is imperative to make a systemic research effort for achieving twin objectives of system productivity and weed control through suitable crop diversification. Present investigation was aimed to evaluate the relative performance of 16 soybean based cropping systems of Malwa Plateau of Madhya Pradesh under assured irrigated production system. These cropping systems were compared for their weed dynamics, system productivity and production efficiency.

\section{Materials and Methods}

A field experiment was conducted for two years during kharif, rabi and zaid seasons of 2015-16 and 2016-17 at Research Farm of Krishi Vigyan Kendra, Dhar (M.P.) located at $22.6013^{\circ} \mathrm{N}$ latitude and $75.3025^{\circ} \mathrm{E}$ longitude with an average altitude of around 588 meters above the mean sea level. Dhar district belongs to "Malwa Plateau" under $10^{\text {th }}$ agroclimatic zone of Madhya Pradesh. Dhar enjoys a typical sub tropical climate consisting 
of hot dry summers and cool dry winters. Temperature extremes vary between a minimum temperature of $12^{0} \mathrm{C}$ in December and January months to maximum temperature of $45^{\circ} \mathrm{C}$ in May and June. The soil of the field was a typical medium black soil. Due to dominance of Montmorillonite clay content it has high capacity to swell and shrink and high CEC. The soil of the experimental field was clay loam in texture, neutral in reaction $(\mathrm{pH}$ 7.60) with normal EC $(0.59 \mathrm{dS} / \mathrm{m})$ and low organic carbon contents $(0.53 \%)$ and low in available $\mathrm{N}(218 \mathrm{~kg} / \mathrm{ha})$, medium in available $\mathrm{P}(11.60 \mathrm{~kg} / \mathrm{ha})$ and high in available K (350 $\mathrm{kg} / \mathrm{ha}$ ) contents. The experiment comprised 16 cropping sequences, soybean was sequenced with feasible rabi viz. Wheat (Triticum aestivum \& Triticum durum L.), Chick pea (Cicer arietinum L.), Garlic (Allium sativum L.), Onion (Allium cepa L.), Potato (Solanum tuberosum L.) and garden pea (Pisum sativum L.) with inclusion of Garlic (Allium sativum L.), Onion (Allium cepa L.) in zaid and tested in randomized block design with four replications.

Only soybean crop was grown during kharif season with two varieties i.e. JS 95-60 early duration (82-87 days) and JS 93-05 medium duration (90-95 days) under all cropsequences, Different varieties were grown under various need based diversified intensive crop sequences as per their feasibility to accommodate the succeeding crop under present investigation, The soybean varieties tested under study were JS 95-60 (a high yielder widely accepted by the farmers in the locality), JS 93-05 (a medium duration high yielding). The variety used for rabi crops was like wheat (HI-1544) aestivum, wheat (HI8663) durum, chickpea (JG-130) desi, chickpea (RVKG-101) kabuli, Potato (Kufri jyoti), garden pea (Arkel) and garlic (G-282) and onion (AFLR) during zaid, respectively. Sowing of kharif, rabi and zaid crops were done in second week of June, October and
February, respectively. Sowing of different crops under different crop sequences was done as per recommended package of practices for crops under irrigated condition.

The recommended dose of $\mathrm{N}: \mathrm{P}: \mathrm{K}(\mathrm{kg} / \mathrm{ha})$ for soybean 20:80:20, wheat 120:60:40, chick pea 20:60:20, garlic 100:50:50, onion 100:75:50, potato 120:50:100 and garden pea 20:60:20 was applied. The nitrogen, phosphorus and potash were applied through urea, single super phosphate and muriate of potash, respectively.

Weeds were controlled in soybean with the use of hand weeding at 20 and 40 DAS. In irrigated wheat, chick pea and garden pea weeds were controlled with the use of hand weeding at 30 DAS. Weed control in onion and garlic was made with the use of hand weeding at 20 and 40 DAT. In potato, weeds were controlled with hand weeding followed by earthing at 20 DAS and with hand weeding only at 50 DAS. After this, weed dynamics of all cropping systems were worked out.

Soybean equivalent yield (SEY) of all cropping systems was also worked out with the help of following formula:

Soybean yield $(\mathrm{SEY})(\mathrm{q} / \mathrm{ha})=$

equivalent Yield of a crop (q/ha) $x$ Price of yield (₹/q)

Price of soybean yield (₹ /q)

The production efficiency (system productivity) of each crop sequence was worked out treatment wise with the help of following formula:

Production efficiency $(\mathrm{kg} / \mathrm{ha} / \mathrm{day})=$

Soybean equivalent yield ( $\mathrm{kg} / \mathrm{ha}$ ) of a particular crop sequences

Total duration of all crop components of the same crop sequence (days) 


\section{Results and Discussion}

\section{Weed dynamics}

The population of different weeds associated with the crops grown under different crop-sequences at 25 DAS and maturity stages was recorded species wise and then their relative density was determined (Table 1).

\section{Relative weed density in kharif season}

Two Soybean varieties were grown in all the 16 crop sequences tested under present investigation during Kharif season. The presence of weed flora was almost similar during both years in all 16 crop-sequences under Soybean varieties. Echinochloa crusgalli was the most dominating weed contributing $27.35 \%$ of total weed intensity at 25 DAT growth stage.

The next predominating weed was Celosia argentea with relative density of $18.15 \%$. The relative density of Commelina benghalensis, Alternanathera triandra, Tridex procumbense, Digera arvensis, and Euphorbia hirta was $18.15,14.15,11.8,8.05,7.25$ and $6.05 \%$ respectively. Remaining minor weeds as a whole had relative density of $7.2 \%$ at 25 DAS. The density of all these weeds changed at maturity stage of soybean. Celosia argentea topped with relative intensity of $28.9 \%$ followed by Alternanathera triandra (16.35 $\%)$, Tridex procumbense (15.85\%), in place of Echinochloa crusgalli (11.7 \%), Digera arvensis $(9.25 \%)$, Commelina benghalensis (6.8\%), Echinochloa crusgalli (11.7\%), Digera arvensis $(9.25 \%)$, and remaining weeds $(3.35 \%)$ at maturity stage of Soybean.

\section{Relative weed density of in Rabi season}

During rabi season, different crops were grown. The relative density of associated weeds in various rabi crops are recorded at 25 DAS and maturity stages (Table 2).

In Wheat crop, the relative density of Chenopodium album, Portulaca oleracea, Phalaris minor, Anagalis arvensis, convolvulus arvensis, Medicago denticulata and Melilotus alba was 26.5, $17.15,12.35,11.0,10.9,8.9$ and $6.55 \%$, respectively and minor weeds contributed to $6.65 \%$ of the total weed density in wheat at 25 DAS. But the relative densities of these weeds including total minor weeds were changed as $24.6,14.35$, $13.15,11.55,10.3,9.25,6.95$ and $9.85 \%$, respectively at maturity stage.

In Chick pea, Chenopodium album, Medicago denticulata, Rumex dentatus, Anagalis arvensis, Portulaca oleracea, convolvulus arvensis, Melilotus alba and other minor weeds contributed 26.75, $14.35,12.65,12.05,10.65,8.15,6.40$ and $9.0 \%$ of the total weed population at 25 DAS, while relative density of these weeds changed as $24.55,15.85,10.90$, $10.50,13.0,8.85,7.7$ and $8.65 \%$, respectively at maturity stage.

In both onion and garlic crops, weed infestation was almost identical. Chenopodium album, Rumex dentatus, Anagallis arvensis, Medicago denticulata, Portulaca oleracea, Melilotus alba, convolvulus arvensis, and other minor weeds contributed to $26.05,12.30,12.05$, $11.9,7.35,6.65$ and $11.65 \%$ of the total weed intensity at 25 DAS in both crops, but the values of relative changed as $21.05,18.50,14.35,13.15,10.60,7.8$, 5.4 , and $9.15 \%$, respectively at maturity stage.

In potato, Cenopodium album, Anagallis arvensis, Portulaca oleracea, Medicago denticulata, Convolvulus arvensis and 
Melilotus alba were the dominating weeds with relative density of $28.35,20.20$, $15.05,11.80, \quad 10.15$ and $7.75 \%$, respectively at 25 DAS, but other minor weeds contributed to $6.75 \%$ in total weed density at this stage. At maturity stage, these weeds contributed to $21.7,8.9,23.7$, $16.4,7.35,10.35$ and $11.6 \%$ of total weeds, respectively.

\section{Relative weed density in zaid season}

During zaid season, onion and garlic crops were grown. The weed infestation was almost identical. Hence, data of weed density of both crops are presented on the basis of mean values of both crops. Cyprus rotundus, Chenopodium album, Anagallis arvensis, convolvulus arvensis, Medicago denticulata, Portulaca oleracea, Melilotus alba, and other minor weeds contributed to $24.05,22.05,14.15$, $11.8,7.40,7.25,6.05$ and $7.25 \%$ of the total weed intensity at 25 DAS in both crops, but the values of relative changed as $26.60,19.2,6.8,15.25,11.75,9.25$, 7.8 , and $3.35 \%$, respectively at maturity stage (Table 3).

\section{Weed intensity}

Data pertaining to total weed intensity at maturity stage of each crop component under various crop sequences during kharif, rabi and zaid seasons are given in Table 4.

Based on 2-year mean data, Soybean (JS 95-60) led to record significant minimum weed intensity $\left(220.0\right.$ and $\left.243.25 / \mathrm{m}^{2}\right)$ and Soybean (JS 93-05) recorded maximum weed intensity $\left(224.2\right.$ and $244.5 / \mathrm{m}^{2}$. Both varieties did not differ much with each other for weed- intensity. During rabi season, weed intensity showed variations due to various crops grown in different crop sequences. The weed population was significantly minimum in $\mathrm{T}_{7}$ - Soybean (JS 95-60)-Potato (Kufri jyoti) Onion (AFLR) i.e. $119.65 \mathrm{dm}^{2}$ closely followed by $\mathrm{T}_{8}$ - Soybean (JS 95-60) - Garden pea (Arkel) - Garlic $121.5 / \mathrm{m}^{2}, \mathrm{~T}_{15^{-}}$Soybean (JS 93-05) - Potato (Kufri jyoti) - Onion (AFLR) $125.95 / \mathrm{m}^{2}$ which was due to diversified and intensified cropping system.

During zaid season, onion significantly allowed maximum infestation of weeds $\left(120.5\right.$ to $\left.121.35 / \mathrm{m}^{2}\right)$ and Garlic allowed minimum infestation of weeds (118.55 to $119.7 / \mathrm{m}^{2}$ ) among both zaid crops.

While comparing the total weedintensity $/ \mathrm{m}^{2}$ for entire crop- cycle of different crop-sequences, $\mathrm{T}_{13}$ - Soybean (JS 93-05) - Garlic (G-282) system had significantly minimum weedintensification $356.40 / \mathrm{m}^{2}$. The weed infestation significantly increased in ascending order as $356.6 / \mathrm{m}^{2}$ in $\mathrm{T}_{14^{-}}$ Soybean (JS 93-05) - Onion (AFLR), $363.75 / \mathrm{m}^{2}$ in $\mathrm{T}_{6}$ - Soybean (JS 95-60) - Onion (AFLR), $366.20 / \mathrm{m}^{2}$ in $\mathrm{T}_{5}$ - Soybean (JS 9560) - Garlic (G-282), $381.10 / \mathrm{m}^{2}$ in $\mathrm{T}_{3^{-}}$ Soybean (JS 95-60) - Chickpea (JG-130) desi, $384.45 / \mathrm{m}^{2}$ in $\mathrm{T}_{4}-$ Soybean (JS 95-60) Chickpea (RVKG-101) Kabuli, $396.85 / \mathrm{m}^{2}$ in $\mathrm{T}_{11}$-Soybean (JS 93-05) - Chickpea (JG-130) desi, 397.05/m $\mathrm{m}^{2}$ in $\mathrm{T}_{12}$-Soybean (JS 93-05) Chickpea (RVKG-101) Kabuli, $399 / \mathrm{m}^{2}$ in $\mathrm{T}_{2-}$ Soybean (JS 95-60) - Wheat (HI-8663) durum, $401.30 / \mathrm{m}^{2}$ in $\mathrm{T}_{9^{-}}$Soybean (JS 93-05) Wheat (HI-1544) aestivum, $407.0 / \mathrm{m}^{2}$ in $\mathrm{T}_{1^{-}}$ Soybean (JS 95-60) - Wheat (HI-1544) aestivum, $461.20 / \mathrm{m}^{2}$ in $\mathrm{T}_{8}$ - Soybean (JS 9560) - Garden pea (Arkel) - Garlic, $473.25 / \mathrm{m}^{2}$ in $\mathrm{T}_{16^{-}}$Soybean (JS 93-05) - Garden pea (Arkel) - Garlic (G-282) and $473.85 / \mathrm{m}^{2}$ in $\mathrm{T}_{7}$ - Soybean (JS 95-60) - Potato (Kufri jyoti) Onion (AFLR), $476.2 / \mathrm{m}^{2}$ in $\mathrm{T}_{15}$ - Soybean (JS 93-05) - Potato (Kufri jyoti) - Onion (AFLR) system. 
But difference between $T_{13}$ and $T_{14} ; T_{5}$, $\mathrm{T}_{6}, \mathrm{~T}_{10}$ and $\mathrm{T}_{11}$ were not significant. As a whole, the crop-sequences with $300 \%$ cropping intensity had higher weed intensity than crop-sequences of $200 \%$ cropping intensity.

\section{Weed biomass}

Data related to weed biomass/ha at maturity stage of each crop under different crop-sequences during kharif, rabi and zaid seasons were recorded (Table 5).

The weed biomass/ha showed little variation due to both Soybean varieties in various crop-sequences during kharif season.

The weed biomass was minimum (15.30 to $27.95 \mathrm{q} / \mathrm{ha}$ ) with Soybean variety JS 95-60 and maximum with Soybean variety JS 93-05 (16.53 to 29.90 q/ha) but variations between JS 95-60 and JS 93-05 were not much.

During rabi season, the weed biomass significantly varied due to different crop components of various crop sequences. The crop sequences consisted with potato led to record significantly lesser weed biomass as $5.35 \mathrm{q} / \mathrm{ha}$ in $\mathrm{T}_{7}$ and $5.50 \mathrm{q} / \mathrm{ha}$ in $\mathrm{T}_{8}$ than remaining crops grown in other crop sequences except to chick pea (6.6 $\mathrm{q} / \mathrm{ha})$ in $\mathrm{T}_{4}$ and garden pea $(6.85 \mathrm{q} / \mathrm{ha})$ in $\mathrm{T}_{16}$.

The weed biomass was maximum (9.45 $\mathrm{q} / \mathrm{ha}$ ) among all rabi crops under $\mathrm{T}_{6}$, which was closely followed by potato $(8.90 \mathrm{q} / \mathrm{ha})$ in $\mathrm{T}_{15}$, wheat $(8.85 \mathrm{q} / \mathrm{ha})$ in $\mathrm{T}_{9}$ and wheat $(8.75 \mathrm{q} / \mathrm{ha})$ in $\mathrm{T}_{10}$. Other rabi crops resulted into reduction of weed biomass ranging from 6.9 to $7.7 \mathrm{q} / \mathrm{ha}$ under $T_{4}, T_{2}$ and $T_{13}$, which were at par to $\mathrm{T}_{14}$ and $\mathrm{T}_{13}$.
The weed biomass also varied significantly due to different zaid crops grown in various crop sequences. Onion under $\mathrm{T}_{15}$ led to record remarkably the highest weed biomass (8.80 q/ha) among zaid crops.

The weed biomass was minimum with garlic $(6.83 \mathrm{q} / \mathrm{ha})$ under $\mathrm{T}_{8}$ closely followed by garlic $(5.75 \mathrm{q} / \mathrm{ha})$ in $\mathrm{T}_{6}$.

While considering the weed biomass of entire cropping system, $\mathrm{T}_{15^{-}}$Soybean (JS 93-05) - Potato (Kufri jyoti) - Onion (AFLR) significantly registered the highest value $28.90 \mathrm{q} / \mathrm{ha}$ ) among all crop sequences, but variations with $\mathrm{T}_{7}$ - Soybean (JS 95-60) Potato (Kufri jyoti) - Onion (AFLR) (24.03 q/ha), $\mathrm{T}_{16^{-}}$Soybean (JS 93-05) - Garden pea (Arkel) - Garlic (G-282) (23.50 q/ha), T (- $^{-}$ Soybean (JS 95-60) -Garden pea (Arkel) Garlic, $\mathrm{T}_{6^{-}}$Soybean (JS 95-60) - Onion (AFLR) $(20.30 \mathrm{q} / \mathrm{ha})$ and $\mathrm{T}_{10^{-}}$Soybean (JS 93-05)-Wheat (HI-8663) durum (19.53 q/ha) were found significant.

The weed biomass was minimum (16.53 $\mathrm{q} /$ ha) with $\mathrm{T}_{14}$-Soybean (JS 93-05)-Onion (AFLR) which was comparable to $\mathrm{T}_{10^{-}}$ Soybean (JS 93-05) - Wheat (HI-8663) durum (19.53 q/ha), $\mathrm{T}_{9-}$ - Soybean (JS 93-05) - Wheat (HI-1544) aestivum (19.33 q/ha), $\mathrm{T}_{2^{-}}$ Soybean (JS 95-60) - Wheat (HI-8663) durum (19.30 q/ha), $\mathrm{T}_{3}$ - Soybean (JS 95-60) Chickpea (JG-130) desi (19.00 q/ha) and $\mathrm{T}_{1^{-}}$ Soybean (JS 95-60) - Wheat (HI-1544) aestivum (18.55 q/ha) and $\mathrm{T}_{5^{-}}$Soybean (JS 95-60) - Garlic (G-282) (18.13q/ha).

The results are in close conformity with Chitale et al., 2011; Walia et al., 2011; Soni et al., 2012; Chander et al., 2013; Jadhav et al., 2014; Pradhan et al., 2014; Punia et al., 2016; Singh et al., 2017; Sethi et al., 2018; Sethi et al., 2019. 


\begin{tabular}{|c|c|c|c|c|c|c|c|}
\hline \multirow[t]{2}{*}{ Crop } & \multirow[t]{2}{*}{ Predominant weeds } & \multicolumn{3}{|c|}{ Relative density (\%) at 25 DAS } & \multicolumn{3}{|c|}{ Relative density (\%) at maturity } \\
\hline & & 2015-16 & 2016-17 & Mean & 2015-16 & 2016-17 & Mean \\
\hline \multirow[t]{9}{*}{ Soybean } & Echinochloa crusgalli & 25.8 & 28.9 & 27.35 & 11.2 & 12.2 & 11.7 \\
\hline & Celosia argentea & 18.5 & 17.8 & 18.15 & 28.6 & 29.2 & 28.9 \\
\hline & Commelina benghalensis & 13.5 & 14.8 & 14.15 & 6.3 & 7.3 & 6.8 \\
\hline & Alternanathera triandra & 12.1 & 11.5 & 11.8 & 17.9 & 14.8 & 16.35 \\
\hline & Tridex procumbense & 8.5 & 7.6 & 8.05 & 16.2 & 15.5 & 15.85 \\
\hline & Digera arvensis & 7.9 & 6.6 & 7.25 & 8.8 & 9.7 & 9.25 \\
\hline & Euphorbia hirta & 5.9 & 6.2 & 6.05 & 7.9 & 7.7 & 7.8 \\
\hline & Others & 7.8 & 6.6 & 7.2 & 3.1 & 3.6 & 3.35 \\
\hline & Total & 100 & 100 & 100 & 100 & 100 & 100 \\
\hline
\end{tabular}

Table.2 Relative density of weeds at 25 DAS and maturity stages of Rabi crops under different crop sequences

\begin{tabular}{|c|c|c|c|c|c|c|c|}
\hline \multirow[t]{2}{*}{ Crop } & \multirow[t]{2}{*}{ Predominant weeds } & \multicolumn{3}{|c|}{ Relative density (\%) at 25 DAS } & \multicolumn{3}{|c|}{ Relative density (\%) at maturity } \\
\hline & & 2015-16 & 2016-17 & Mean & 2015-16 & 2016-17 & Mean \\
\hline \multirow[t]{9}{*}{ Wheat } & Chenopodium album & 26.5 & 26.5 & 26.5 & 25.4 & 23.8 & 24.6 \\
\hline & Portulaca oleracea & 16.8 & 17.5 & 17.15 & 13.9 & 14.8 & 14.35 \\
\hline & Phalaris minor & 12.8 & 11.9 & 12.35 & 13.5 & 12.8 & 13.15 \\
\hline & Convolvulus arvensis & 10.6 & 11.2 & 10.9 & 11.3 & 11.8 & 11.55 \\
\hline & Anagalis arvensis & 10.5 & 11.5 & 11 & 10.7 & 9.9 & 10.3 \\
\hline & Medicago denticulata & 9.5 & 8.3 & 8.9 & 8.8 & 9.7 & 9.25 \\
\hline & Melilotus alba & 6.8 & 6.3 & 6.55 & 6.6 & 7.3 & 6.95 \\
\hline & Others & 6.5 & 6.8 & 6.65 & 9.8 & 9.9 & 9.85 \\
\hline & Total & 100 & 100 & 100 & 100 & 100 & 100 \\
\hline
\end{tabular}




\begin{tabular}{|c|c|c|c|c|c|c|c|}
\hline \multirow[t]{2}{*}{ Crop } & \multirow[t]{2}{*}{ Predominant weeds } & \multicolumn{3}{|c|}{ Relative density (\%) at 25 DAS } & \multicolumn{3}{|c|}{ Relative density (\%) at maturity } \\
\hline & & 2015-16 & 2016-17 & Mean & 2015-16 & 2016-17 & Mean \\
\hline \multirow[t]{9}{*}{ Chick pea } & Chenopodium album & 27.6 & 25.9 & 26.75 & 25.2 & 23.9 & 24.55 \\
\hline & Medicago denticulata & 13.9 & 14.8 & 14.35 & 16.2 & 15.5 & 15.85 \\
\hline & Rumex dentatus & 12.5 & 12.8 & 12.65 & 10.6 & 11.2 & 10.9 \\
\hline & Anagalis arvensis & 11.6 & 12.5 & 12.05 & 9.8 & 11.2 & 10.5 \\
\hline & Portulaca oleracea & 10.8 & 10.5 & 10.65 & 12.5 & 13.5 & 13 \\
\hline & Melilotus alba & 6.9 & 5.9 & 6.4 & 8.8 & 8.9 & 8.85 \\
\hline & Convolvulus arvensis & 8.5 & 7.8 & 8.15 & 7.8 & 7.6 & 7.7 \\
\hline & Others & 8.2 & 9.8 & 9 & 9.1 & 8.2 & 8.65 \\
\hline & Total & 100 & 100 & 100 & 100 & 100 & 100 \\
\hline \multirow[t]{2}{*}{ Crop } & \multirow[t]{2}{*}{ Predominant weeds } & \multicolumn{3}{|c|}{ Relative density (\%) at 25 DAS } & \multicolumn{3}{|c|}{ Relative density (\%) at maturity } \\
\hline & & 2015-16 & 2016-17 & Mean & 2015-16 & 2016-17 & Mean \\
\hline \multirow[t]{9}{*}{ Onion \& Garlic } & Chenopodium album & 26.2 & 25.9 & 26.05 & 22.3 & 19.8 & 21.05 \\
\hline & Rumex dentatus & 11.8 & 12.8 & 12.30 & 18.1 & 18.9 & 18.50 \\
\hline & Anagalis arvensis & 11.6 & 12.5 & 12.05 & 13.9 & 14.8 & 14.35 \\
\hline & Medicago denticulata & 12.3 & 11.8 & 12.05 & 13.5 & 12.8 & 13.15 \\
\hline & Portulaca oleracea & 11.5 & 12.3 & 11.9 & 10.6 & 10.6 & 10.6 \\
\hline & Melilotus alba & 8.8 & 5.9 & 7.35 & 7.9 & 7.7 & 7.8 \\
\hline & Convolvulus arvensis & 5.5 & 7.8 & 6.65 & 5.2 & 5.6 & 5.4 \\
\hline & Others & 12.3 & 11 & 11.65 & 8.5 & 9.8 & 9.15 \\
\hline & Total & 100 & 100 & 100 & 100 & 100 & 100 \\
\hline
\end{tabular}




\begin{tabular}{|c|c|c|c|c|c|c|c|}
\hline \multirow[t]{2}{*}{ Crop } & \multirow[t]{2}{*}{ Predominant weeds } & \multicolumn{3}{|c|}{ Relative density (\%) at 25 DAS } & \multicolumn{3}{|c|}{ Relative density (\%) at maturity } \\
\hline & & 2015-16 & 2016-17 & Mean & 2015-16 & 2016-17 & Mean \\
\hline \multirow[t]{8}{*}{ Potato } & Chenopodium album & 27.8 & 28.9 & 28.35 & 21.2 & 22.2 & 21.7 \\
\hline & Anagalis arvensis & 22.6 & 17.8 & 20.2 & 8.6 & 9.2 & 8.9 \\
\hline & Portulaca oleracea & 12.6 & 17.5 & 15.05 & 26.2 & 21.2 & 23.7 \\
\hline & Medicago denticulata & 12.1 & 11.5 & 11.8 & 17 & 15.8 & 16.4 \\
\hline & Convolvulus arvensis & 9.8 & 10.5 & 10.15 & 6.3 & 8.4 & 7.35 \\
\hline & Melilotus alba & 7.9 & 7.6 & 7.75 & 9.9 & 10.8 & 10.35 \\
\hline & Others & 7.2 & 6.2 & 6.7 & 10.8 & 12.4 & 11.6 \\
\hline & Total & 100 & 100 & 100 & 100 & 100 & 100 \\
\hline \multirow[t]{2}{*}{ Crop } & \multirow[t]{2}{*}{ Predominant weeds } & \multicolumn{3}{|c|}{ Relative density (\%) at 25 DAS } & \multicolumn{3}{|c|}{ Relative density (\%) at maturity } \\
\hline & & 2015-16 & 2016-17 & Mean & 2015-16 & 2016-17 & Mean \\
\hline \multirow[t]{7}{*}{ Garden pea } & Chenopodium album & 35.40 & 36.8 & 36.10 & 22.2 & 21.4 & 21.80 \\
\hline & Anagalis arvensis & 23.50 & 24.8 & 24.15 & 16.3 & 17.8 & 17.05 \\
\hline & Portulaca oleracea & 17.40 & 15.5 & 16.45 & 24.9 & 22.4 & 23.65 \\
\hline & Medicago denticulata & 8.40 & 7.6 & 8.00 & 16.2 & 15.5 & 15.85 \\
\hline & Melilotus alba & 6.30 & 6.8 & 6.55 & 12.3 & 13.9 & 13.10 \\
\hline & Others & 9.00 & 8.5 & 8.75 & 8.1 & 9.00 & 8.55 \\
\hline & Total & 100 & 100 & 100 & 100 & 100 & 100 \\
\hline
\end{tabular}


Int.J.Curr.Microbiol.App.Sci (2019) 8(10): 1626-1643

\begin{tabular}{|c|c|c|c|c|c|c|c|}
\hline \multirow[t]{2}{*}{ Crop } & \multirow[t]{2}{*}{ Predominant weeds } & \multicolumn{3}{|c|}{ Relative density (\%) at 25 DAS } & \multicolumn{3}{|c|}{ Relative density (\%) at maturity } \\
\hline & & 2015-16 & 2016-17 & Mean & 2015-16 & 2016-17 & Mean \\
\hline \multirow{9}{*}{$\begin{array}{c}\text { Onion \& } \\
\text { Garlic }\end{array}$} & Cyprus rotundus & 23.5 & 24.6 & 24.05 & 28 & 25.2 & 26.6 \\
\hline & Chenopodium album & 22.3 & 21.8 & 22.05 & 19.8 & 18.6 & 19.2 \\
\hline & Anagalis arvensis & 13.5 & 14.8 & 14.15 & 6.3 & 7.3 & 6.8 \\
\hline & Convolvulus arvensis & 12.1 & 11.5 & 11.8 & 17.9 & 12.6 & 15.25 \\
\hline & Medicago denticulata & 7.6 & 7.2 & 7.4 & 8.2 & 15.3 & 11.75 \\
\hline & Portulaca oleracea & 7.9 & 6.6 & 7.25 & 8.8 & 9.7 & 9.25 \\
\hline & Melilotus alba & 5.9 & 6.2 & 6.05 & 7.9 & 7.7 & 7.8 \\
\hline & Others & 7.2 & 7.3 & 7.25 & 3.1 & 3.6 & 3.35 \\
\hline & Total & 100 & 100 & 100 & 100 & 100 & 100 \\
\hline
\end{tabular}


Table.4 Total weed intensity $/ \mathrm{m}^{2}$ at maturity stage under different crop sequences

\begin{tabular}{|c|c|c|c|c|c|c|c|c|c|c|c|}
\hline \multicolumn{2}{|r|}{ Crop sequences } & \multicolumn{3}{|c|}{ Kharif } & \multicolumn{3}{|c|}{ Rabi } & \multicolumn{3}{|c|}{ Zaid } & \multirow{2}{*}{$\begin{array}{l}\text { Crop } \\
\text { cycle }\end{array}$} \\
\hline & & $\begin{array}{c}2015- \\
16\end{array}$ & $\begin{array}{c}2016- \\
17\end{array}$ & Mean & $\begin{array}{l}\text { 2015- } \\
16\end{array}$ & $\begin{array}{c}2016- \\
17\end{array}$ & Mean & $\begin{array}{c}2015- \\
16\end{array}$ & $\begin{array}{c}2016- \\
17\end{array}$ & Mean & \\
\hline $\mathbf{T}_{1}$ & Soybean (JS 95-60) - Wheat (HI-1544) aestivum & 242.20 & 236.80 & 239.5 & 166.50 & 168.50 & 167.5 & - & - & - & 407.00 \\
\hline $\mathbf{T}_{2}$ & Soybean (JS 95-60) - Wheat (HI-8663) durum & 243.50 & 237.50 & 240.5 & 155.80 & 161.20 & 158.5 & - & - & - & 399.00 \\
\hline $\mathbf{T}_{\mathbf{3}}$ & Soybean (JS 95-60) - Chickpea (JG-130) desi & 248.50 & 238.00 & 243.25 & 135.20 & 140.50 & 137.85 & - & - & - & 381.10 \\
\hline $\mathbf{T}_{4}$ & Soybean (JS 95-60) - Chickpea (RVKG-101) Kabuli & 244.60 & 238.60 & 241.6 & 142.50 & 143.20 & 142.85 & - & - & - & 384.45 \\
\hline $\mathbf{T}_{5}$ & Soybean (JS 95-60) - Garlic (G-282) & 237.50 & 240.20 & 238.85 & 126.90 & 127.80 & 127.35 & - & - & - & 366.20 \\
\hline $\mathbf{T}_{6}$ & Soybean (JS 95-60) - Onion (AFLR) & 233.60 & 236.50 & 235.05 & 128.90 & 128.50 & 128.7 & - & - & - & 363.75 \\
\hline $\mathbf{T}_{7}$ & $\begin{array}{c}\text { Soybean (JS 95-60) - Potato (Kufri jyoti) - Onion } \\
\text { (AFLR) }\end{array}$ & 226.50 & 239.20 & 232.85 & 118.10 & 121.20 & 119.65 & 122.90 & 119.80 & 121.35 & 473.85 \\
\hline $\mathbf{T}_{8}$ & $\begin{array}{c}\text { Soybean (JS 95-60) - Garden pea (Arkel) - Garlic } \\
\text { (G-282) }\end{array}$ & 219.50 & 220.50 & 220 & 120.50 & 122.50 & 121.5 & 122.60 & 116.80 & 119.7 & 461.20 \\
\hline $\mathbf{T}_{9}$ & Soybean (JS 93-05) - Wheat (HI-1544) aestivum & 246.30 & 239.20 & 242.75 & 156.90 & 160.20 & 158.55 & - & - & - & 401.30 \\
\hline $\mathbf{T}_{10}$ & Soybean (JS 93-05) - Wheat (HI-8663) durum & 241.20 & 239.00 & 240.1 & 154.20 & 161.20 & 157.7 & - & - & - & 397.80 \\
\hline $\mathbf{T}_{11}$ & Soybean (JS 93-05) - Chickpea (JG-130) desi & 247.50 & 241.50 & 244.5 & 149.50 & 155.20 & 152.35 & - & - & - & 396.85 \\
\hline $\mathbf{T}_{12}$ & Soybean (JS 93-05) - Chickpea (RVKG-101) Kabuli & 244.30 & 238.50 & 241.4 & 155.00 & 156.30 & 155.65 & - & - & - & 397.05 \\
\hline $\mathbf{T}_{13}$ & Soybean (JS 93-05) - Garlic (G-282) & 229.30 & 226.50 & 227.9 & 129.20 & 127.80 & 128.5 & - & - & - & 356.40 \\
\hline $\mathbf{T}_{14}$ & Soybean (JS 93-05) - Onion (AFLR) & 230.50 & 225.80 & 228.15 & 127.40 & 129.50 & 128.45 & - & - & - & 356.60 \\
\hline $\mathbf{T}_{15}$ & $\begin{array}{c}\text { Soybean (JS 93-05) - Potato (Kufri jyoti) - Onion } \\
\text { (AFLR) }\end{array}$ & 229.00 & 230.50 & 229.75 & 125.40 & 126.50 & 125.95 & 121.50 & 119.50 & 120.5 & 476.20 \\
\hline $\mathbf{T}_{16}$ & $\begin{array}{c}\text { Soybean (JS 93-05) - Garden pea (Arkel) - Garlic } \\
\text { (G-282) }\end{array}$ & 227.80 & 223.60 & 225.7 & 128.50 & 129.50 & 129 & 119.60 & 117.50 & 118.55 & 473.25 \\
\hline & SEm \pm & 1.59 & 1.69 & 1.16 & 1.12 & 1.98 & 1.14 & 1.21 & 1.46 & 0.95 & 1.853 \\
\hline & $\mathrm{CD}(\mathrm{P}=\mathbf{0 . 0 5})$ & 4.55 & 4.84 & 3.25 & 3.20 & 5.65 & 3.18 & 3.45 & 4.17 & 2.65 & 5.296 \\
\hline
\end{tabular}




\begin{tabular}{|c|c|c|c|c|c|c|c|c|c|c|c|}
\hline \multicolumn{12}{|c|}{ Table.5 Total weed biomass ( $\mathrm{q} / \mathrm{ha}$ ) at maturity stage under different crop sequences } \\
\hline \multirow{2}{*}{\multicolumn{2}{|c|}{ Crop sequences }} & \multirow{2}{*}{\multicolumn{3}{|c|}{ Kharif }} & \multirow{2}{*}{\multicolumn{3}{|c|}{ Rabi }} & \multirow{2}{*}{\multicolumn{3}{|c|}{ Zaid }} & \multirow{3}{*}{$\begin{array}{l}\begin{array}{l}\text { Crop } \\
\text { cycle }\end{array} \\
18.55\end{array}$} \\
\hline & & & & \multirow{2}{*}{\begin{tabular}{|l|} 
Mean \\
10.85
\end{tabular}} & & & & & & & \\
\hline$T_{1}$ & Soybean (JS 95-60) - Wheat (HI-1544) aestivum & $\begin{array}{c}\text { 2015-16 } \\
11.50\end{array}$ & $\begin{array}{c}\text { 2016-17 } \\
10.20\end{array}$ & & $\begin{array}{c}\text { 2015-16 } \\
8.89\end{array}$ & $\begin{array}{c}\text { 2016-17 } \\
6.50\end{array}$ & $\begin{array}{c}\text { Mean } \\
7.70\end{array}$ & $\begin{array}{c}\text { 2015- } \\
16 \\
-\end{array}$ & $\begin{array}{c}2016- \\
17 \\
-\end{array}$ & $\begin{array}{c}\text { Mean } \\
-\end{array}$ & \\
\hline $\mathbf{T}_{2}$ & Soybean (JS 95-60) - Wheat (HI-8663) durum & 12.50 & 11.40 & 11.95 & 7.80 & 6.90 & 7.35 & - & - & - & 1930 \\
\hline $\mathbf{T}_{3}$ & Soybean (JS 95-60) - Chickpea (JG-130) desi & 12.10 & 10.90 & 11.50 & 8.20 & 6.80 & 7.50 & - & - & - & 19.00 \\
\hline $\mathbf{T}_{4}$ & Soybean (JS 95-60) - Chickpea (RVKG-101) Kabuli & 10.12 & 10.12 & 10.12 & 7.00 & 6.20 & 6.60 & - & - & - & 16.72 \\
\hline $\mathbf{T}_{5}$ & Soybean (JS 95-60) - Garlic (G-282) & 11.45 & 10.40 & 10.93 & 8.50 & 5.90 & 7.20 & - & - & - & 18.13 \\
\hline $\mathbf{T}_{6}$ & Soybean (JS 95-60) - Onion (AFLR) & 11.35 & 10.35 & 10.85 & 9.10 & 9.80 & 9.45 & - & - & - & 20.30 \\
\hline $\mathbf{T}_{7}$ & $\begin{array}{c}\text { Soybean (JS 95-60) - Potato (Kufri jyoti) - Onion } \\
\text { (AFLR) }\end{array}$ & 10.76 & 9.10 & 9.93 & 5.50 & 5.20 & 5.35 & 8.2 & 9.3 & 8.75 & 24.03 \\
\hline $\mathbf{T}_{8}$ & $\begin{array}{l}\text { Soybean (JS 95-60) - Garden pea (Arkel) - Garlic (G- } \\
\text { 282) }\end{array}$ & 10.40 & 9.20 & 9.80 & 5.70 & 5.30 & 5.50 & 6.9 & 6.75 & 6.83 & 22.13 \\
\hline $\mathbf{T}_{9}$ & Soybean (JS 93-05) - Wheat (HI-1544) aestivum & 10.56 & 10.40 & 10.48 & 9.10 & 8.60 & 8.85 & - & - & - & 19.33 \\
\hline $\mathbf{T}_{10}$ & Soybean (JS 93-05) - Wheat (HI-8663) durum & 11.05 & 10.50 & 10.78 & 8.80 & 8.70 & 8.75 & - & - & - & 19.53 \\
\hline $\mathbf{T}_{11}$ & Soybean (JS 93-05) - Chickpea (JG-130) desi & 10.50 & 10.20 & 10.35 & 8.20 & 6.60 & 7.40 & - & - & - & 17.75 \\
\hline $\mathbf{T}_{12}$ & Soybean (JS 93-05) - Chickpea (RVKG-101) Kabuli & 11.65 & 10.40 & 11.03 & 7.00 & 6.80 & 6.90 & - & - & - & 17.93 \\
\hline $\mathbf{T}_{13}$ & Soybean (JS 93-05) - Garlic (G-282) & 9.27 & 9.80 & 9.535 & 8.50 & 5.80 & 7.15 & - & - & - & 16.69 \\
\hline $\mathbf{T}_{14}$ & Soybean (JS 93-05) - Onion (AFLR) & 9.20 & 9.25 & 9.225 & 9.10 & 5.50 & 7.30 & - & - & - & 16.53 \\
\hline $\mathbf{T}_{15}$ & $\begin{array}{c}\text { Soybean (JS 93-05) - Potato (Kufri jyoti) - Onion } \\
\text { (AFLR) }\end{array}$ & 12.20 & 13.10 & 12.65 & 8.50 & 9.30 & 8.90 & 6.90 & 7.80 & 7.35 & 28.90 \\
\hline \multirow[t]{3}{*}{$\mathbf{T}_{16}$} & $\begin{array}{l}\text { Soybean (JS 93-05) - Garden pea (Arkel) - Garlic (G- } \\
\text { 282) }\end{array}$ & 10.80 & 11.00 & 10.9 & 6.70 & 7.00 & 6.85 & 5.60 & 5.90 & 5.75 & 23.50 \\
\hline & SEm \pm & 0.45 & 0.48 & $\mathbf{0 . 3 3}$ & 0.37 & 0.45 & 0.29 & 0.17 & 0.23 & 0.14 & 0.523 \\
\hline & CD $(\mathbf{P}=\mathbf{0 . 0 5})$ & 1.28 & 1.37 & 0.92 & 1.05 & 1.28 & 0.81 & 0.49 & 0.66 & 0.40 & 1.495 \\
\hline & & & & & & & & & & & \\
\hline
\end{tabular}




\begin{tabular}{|c|c|c|c|c|c|c|c|c|c|c|}
\hline \multicolumn{11}{|c|}{ Table.6 Mean Economic yield (q/ha) in different seasons under various crop sequences } \\
\hline \multirow{2}{*}{\multicolumn{2}{|c|}{ Crop sequences }} & \multicolumn{3}{|c|}{ Kharif } & \multicolumn{3}{|c|}{ Rabi } & \multicolumn{3}{|c|}{ Zaid } \\
\hline & & \multirow{2}{*}{$\begin{array}{c}\mathbf{2 0 1 5}-\mathbf{1 6} \\
18.41\end{array}$} & \multirow{2}{*}{$\begin{array}{c}\mathbf{2 0 1 6 - 1 7} \\
19.6\end{array}$} & \multirow{2}{*}{$\begin{array}{c}\text { Mean } \\
19.01\end{array}$} & \multirow{2}{*}{$\begin{array}{c}\mathbf{2 0 1 5 - 1 6} \\
45.83\end{array}$} & \multirow{2}{*}{$\begin{array}{c}\text { 2016-17 } \\
49.3\end{array}$} & \multirow{2}{*}{$\begin{array}{l}\text { Mean } \\
47.57\end{array}$} & \multirow{2}{*}{$\begin{array}{c}2015-16 \\
-\end{array}$} & \multirow{2}{*}{$\begin{array}{c}\text { 2016-17 } \\
-\end{array}$} & \multirow{2}{*}{$\begin{array}{c}\text { Mean } \\
-\end{array}$} \\
\hline $\mathbf{T}_{1}$ & Soybean (JS 95-60) - Wheat (HI-1544) aestivum & & & & & & & & & \\
\hline $\mathbf{T}_{2}$ & Soybean (JS 95-60) - Wheat (HI-8663) durum & 18.48 & 19.87 & 19.18 & 49.4 & 53.05 & 51.23 & - & - & - \\
\hline $\mathbf{T}_{3}$ & Soybean (JS 95-60) - Chickpea (JG-130) desi & 18.81 & 20.11 & 19.46 & 15.8 & 16.95 & 16.38 & - & - & - \\
\hline $\mathbf{T}_{4}$ & $\begin{array}{c}\text { Soybean (JS 95-60) - Chickpea (RVKG-101) } \\
\text { Kabuli }\end{array}$ & 19.05 & 19.03 & 19.04 & 17.23 & 18.68 & 17.96 & - & - & - \\
\hline $\mathbf{T}_{5}$ & Soybean (JS 95-60) - Garlic (G-282) & 19.37 & 20.13 & 19.75 & 83.43 & 84.75 & 84.09 & - & - & - \\
\hline $\mathbf{T}_{6}$ & Soybean (JS 95-60) - Onion (AFLR) & 19.3 & 20.22 & 19.76 & 214 & 226.18 & 220.09 & - & - & - \\
\hline $\mathbf{T}_{7}$ & $\begin{array}{c}\text { Soybean (JS 95-60) - Potato (Kufri jyoti) - Onion } \\
\text { (AFLR) }\end{array}$ & 19.58 & 20.78 & 20.18 & 179.68 & 194.15 & 186.92 & 185.56 & 192.60 & 189.08 \\
\hline $\mathbf{T}_{8}$ & $\begin{array}{c}\text { Soybean (JS 95-60) - Garden pea (Arkel) - Garlic } \\
\text { (G-282) }\end{array}$ & 20.02 & 20.46 & 20.24 & 11.6 & 12.58 & 12.09 & 68.40 & 71.75 & 70.08 \\
\hline $\mathbf{T}_{9}$ & Soybean (JS 93-05) - Wheat (HI-1544) aestivum & 20.43 & 21.25 & 20.84 & 47.88 & 47.73 & 47.81 & - & - & - \\
\hline $\mathbf{T}_{10}$ & Soybean (JS 93-05) - Wheat (HI-8663) durum & 20.17 & 21.08 & 20.63 & 51.23 & 51.73 & 51.48 & - & - & - \\
\hline $\mathbf{T}_{11}$ & Soybean (JS 93-05) - Chickpea (JG-130) desi & 19.69 & 21.44 & 20.57 & 16.9 & 17.3 & 17.10 & - & - & - \\
\hline $\mathbf{T}_{12}$ & $\begin{array}{c}\text { Soybean (JS 93-05) - Chickpea (RVKG-101) } \\
\text { Kabuli }\end{array}$ & 19.41 & 21.35 & 20.38 & 18.65 & 19.71 & 19.18 & - & - & - \\
\hline $\mathbf{T}_{13}$ & Soybean (JS 93-05) - Garlic (G-282) & 20.04 & 22.33 & 21.19 & 85.9 & 86.8 & 86.35 & - & - & - \\
\hline $\mathbf{T}_{14}$ & Soybean (JS 93-05) - Onion (AFLR) & 20.31 & 22.85 & 21.58 & 217.8 & 228.5 & 223.15 & - & - & - \\
\hline $\mathbf{T}_{15}$ & $\begin{array}{c}\text { Soybean (JS 93-05) - Potato (Kufri jyoti) - Onion } \\
\text { (AFLR) }\end{array}$ & 19.67 & 22.9 & 21.29 & 182.5 & 198.3 & 190.40 & 193.48 & 197.85 & 195.67 \\
\hline $\mathbf{T}_{16}$ & $\begin{array}{c}\text { Soybean (JS 93-05) - Garden pea (Arkel) - Garlic } \\
\text { (G-282) }\end{array}$ & 20.65 & 22.48 & 21.57 & 12.3 & 13.25 & 12.78 & 70.65 & 73.60 & 72.13 \\
\hline
\end{tabular}


Table.7 Soybean Equivalent Yield and production efficiency in different seasons under various crop sequences

\begin{tabular}{|c|c|c|c|c|c|c|c|}
\hline \multicolumn{2}{|r|}{ Crop sequences } & \multicolumn{3}{|c|}{ SEY (q/ha) } & \multicolumn{3}{|c|}{ Production efficiency (kg/ha/day) } \\
\hline & & 2015-16 & 2016-17 & Pooled & 2015-16 & 2016-17 & Pooled \\
\hline $\mathbf{T}_{1}$ & Soybean (JS 95-60) - Wheat (HI-1544) aestivum & 47.55 & 50.63 & 49.09 & 22.64 & 23.22 & 22.93 \\
\hline $\mathbf{T}_{2}$ & Soybean (JS 95-60) - Wheat (HI-8663) durum & 49.39 & 52.95 & 51.17 & 23.19 & 23.99 & 23.59 \\
\hline $\mathbf{T}_{\mathbf{3}}$ & Soybean (JS 95-60) - Chickpea (JG-130) desi & 41.46 & 44.11 & 42.79 & 20.56 & 20.33 & 20.44 \\
\hline $\mathbf{T}_{4}$ & Soybean (JS 95-60) - Chickpea (RVKG-101) Kabuli & 46.73 & 48.93 & 47.83 & 23.37 & 23.20 & 23.28 \\
\hline $\mathbf{T}_{5}$ & Soybean (JS 95-60) - Garlic (G-282) & 81.17 & 82.90 & 82.03 & 34.69 & 33.98 & 34.33 \\
\hline $\mathbf{T}_{6}$ & Soybean (JS 95-60) - Onion (AFLR) & 98.55 & 103.92 & 101.24 & 43.23 & 43.52 & 43.37 \\
\hline $\mathbf{T}_{7}$ & Soybean (JS 95-60) - Potato (Kufri jyoti) - Onion (AFLR) & 168.17 & 178.20 & 173.18 & 56.24 & 56.56 & 56.40 \\
\hline $\mathbf{T}_{8}$ & Soybean (JS 95-60) - Garden pea (Arkel) - Garlic (G-282) & 87.88 & 92.24 & 90.06 & 31.50 & 32.03 & 31.77 \\
\hline $\mathbf{T}_{\mathbf{9}}$ & Soybean (JS 93-05) - Wheat (HI-1544) aestivum & 51.34 & 54.33 & 52.83 & 23.77 & 24.47 & 24.12 \\
\hline $\mathbf{T}_{10}$ & Soybean (JS 93-05) - Wheat (HI-8663) durum & 52.77 & 55.88 & 54.33 & 24.32 & 24.95 & 24.63 \\
\hline $\mathbf{T}_{11}$ & Soybean (JS 93-05) - Chickpea (JG-130) desi & 43.19 & 46.44 & 44.81 & 21.07 & 21.49 & 21.28 \\
\hline $\mathbf{T}_{12}$ & Soybean (JS 93-05) - Chickpea (RVKG-101) Kabuli & 47.91 & 51.85 & 49.88 & 22.26 & 24.12 & 23.19 \\
\hline $\mathbf{T}_{13}$ & Soybean (JS 93-05) - Garlic (G-282) & 82.54 & 85.83 & 84.19 & 34.83 & 34.75 & 34.79 \\
\hline $\mathbf{T}_{14}$ & Soybean (JS 93-05) - Onion (AFLR) & 100.81 & 108.05 & 104.43 & 44.07 & 44.65 & 44.36 \\
\hline $\mathbf{T}_{15}$ & Soybean (JS 93-05) - Potato (Kufri jyoti) - Onion (AFLR) & 172.15 & 182.47 & 177.31 & 56.08 & 57.02 & 56.55 \\
\hline $\mathbf{T}_{16}$ & Soybean (JS 93-05) - Garden pea (Arkel) - Garlic (G-282) & 90.18 & 96.70 & 93.44 & 31.64 & 33.01 & 32.33 \\
\hline & SEm \pm & 1.41 & 1.75 & 1.12 & 0.84 & 0.68 & 0.54 \\
\hline & $\mathrm{CD}(\mathrm{P}=0.05)$ & 4.04 & 5.00 & 3.15 & 2.40 & 1.94 & 1.51 \\
\hline
\end{tabular}




\section{System Productivity}

Soybean Yield Equivalent (SEY) of cropping-system as a whole, $\mathrm{T}_{15}$-Soybean (JS 93-05)-Potato (Kufri jyoti)-Onion (AFLR) system was recorded significantly maximum SEYs (177.31 q/ha) among all crop-sequences mainly due to greater SEY of potato during Rabi along with considering good SEYs of onion in zaid season. The next best crop- sequence was $\mathrm{T}_{7}$ - Soybean (JS 95-60)-Potato (Kufri jyoti)Onion (AFLR) with regard to SEYs $(173.18 \mathrm{q} / \mathrm{ha})$ mainly owing to the higher SEYs in kharif soybean and rabi potato and onion in zaid followed by SEY $104.43 \mathrm{q} / \mathrm{ha}$ in $\mathrm{T}_{14-}$ Soybean (JS 93-05) Onion (AFLR), SEY $101.24 \mathrm{q} / \mathrm{ha}$ in $\mathrm{T}_{6^{-}}$ Soybean (JS 95-60) - Onion (AFLR), SEY $93.44 \mathrm{q} / \mathrm{ha}$ in $\mathrm{T}_{16^{-}}$Soybean (JS 93-05) Garden pea (Arkel) - Garlic (G-282) and SEY $90.06 \mathrm{q} / \mathrm{ha}$ in $\mathrm{T}_{8-}$ - Soybean (JS 95-60) - Garden pea (Arkel) - Garlic (G-282). The higher SEYs in Soybean (JS 93-05) - Onion (AFLR) and Soybean (JS 95-60) - Onion (AFLR) cropping sequences was become of higher yield of onion in the sequence. Further, the results revealed that there is sufficient scope to intensify the existing cropping sequence with inclusion of onion and garlic during zaid. Inclusion of onion and garlic during zaid increased cropping sequence productivity. The minimum productivity of the cropping sequence based on SEYs was registered in Soybean (JS 95-60) - Chickpea (JG-130) desi i.e. 42.79 $\mathrm{q} / \mathrm{ha}$. This could be ascribed due to low yield realized from desi chick pea in the sequence. Several researchers have also reported heterogeneity in production of potential varying crop-sequences from different agro- production systems (Chitale et al., 2011; Narkhede, et al., 2011; Kumar, et al., 2012; Billore 2013, Gallani et al., 2013, Shrikant et al., 2013; Prajapat et al., 2014; Singh and Kumar, 2014; Shridhara et. al., 2017; Singh et al., 2017; Jugnahake et al., 2018).

\section{Production Efficiency}

Production efficiency of crop (s)/cropping system(s) refers to economic yields realized from a unit area of land during a unit time needed to grow any crop(s)/cropping system(s) successfully (Table 6 and 7).

Among different crop-sequences tested, $\mathrm{T}_{15^{-}}$Soybean (JS 93-05) - Potato (Kufri jyoti) - Onion (AFLR) registered significantly highest production efficiency $(56.55 \mathrm{~kg} / \mathrm{ha} /$ day $)$. The productivity of potato was found maximum during rabi season followed by onion in zaid season. Thus, this crop sequence produced maximum SEY (177.31 q/ha), through the crop duration for this sequence was higher than other crop sequences, which attributed to maximum production efficiency. The next best crop-sequence was $\mathrm{T}_{7}$-Soybean (JS 95-60)-Potato (Kufri jyoti)-Onion (AFLR) with significantly higher production efficiency of (56.40 $\mathrm{kg} / \mathrm{ha} /$ day), closely followed by $\mathrm{T}_{14^{-}}$ Soybean (JS 93-05)-Onion (AFLR) (44.36 $\mathrm{kg} / \mathrm{ha} /$ day) and $\mathrm{T}_{6-}$ - Soybean (JS 95-60)Onion (AFLR) (43.37 kg/ha/day). The superiority of the production efficiency was due to relatively higher SEY from potato and onion crops during rabi season and inclusion of onion during Zaid under these two cropping systems. Remaining diversified intensive crop sequences led to record production efficiency ranging from 20.44 to $34.79 \mathrm{~kg} / \mathrm{ha} / \mathrm{day}$, which were higher than existing cropping systems viz, soybean-chickpea $(23.59 \mathrm{~kg} / \mathrm{ha} /$ day $)$ and soybean -wheat $(22.93 \mathrm{~kg} / \mathrm{ha} /$ day $)$ systems. Garlic being a high yielding crop during Rabi season in succession to soybean crop with both varieties (JS 95- 
60 and JS 93-05) having high market value under garlic system led to record handsome production efficiency of 34.33 and $34.79 \mathrm{~kg} / \mathrm{ha} /$ day, respectively. Thus, it is evident from the foregoing results that the production efficiency of diversified intensive cropping systems with 300\% cropping intensity could be enhanced over prevailing cropping systems with $200 \%$ cropping intensity. It is also remarkable here that replacement of wheat or chickpea by substituting high value crops likes potato, onion, garlic, durum wheat and kabuli chick pea along with seed production gave higher production efficiency than existing cropping systems, when it's cropping intensity was only $200 \%$. It gave an idea to belief that diversification of traditional crops with other high value crops has an opportunity to enhance the productivity and production efficiency of cropping system. Similar high values of production efficiencies with the inclusion of high yielding crops under diversified intensive cropping systems have been also reported by several other workers from the studies made under varying agro- climatic conditions (Sharma et al., 2008; Chitle et al., 2011; Narkhede, et al., 2011; Tyagi et al., 2011; Billore, 2013; Gallani et al., 2013; Shrikant et al., 2013; Prajapat et al., 2014; Shridhara et al., 2017, Jugnahake et al., 2018).

It can be concluded that relative weed density and weed-flora differed from crop to crop from early stage to maturity of crops. Thus, infestation of severe weeds viz., Echinochloa crusgalli and Celosia argentea in soybean, Chenopodium album and Portulaca oleracea in wheat, Chenopodium album, Medicago denticulate in Chick pea, Chenopodium album and Rumex dentatus in onion and garlic crops, Cenopodium album and Anagallis arvensis in potato could be minimized by intensified and diversified them with other crops with higher system productivity and production efficiency.

\section{References}

Billore, S. D., Ramesh, A., Joshi, O. P. and Vyas, A. K.,2005. Influence of tillage operations on sustainable production of soybean based cropping systems. Soybean Research; 3 (17): 22-15.

Billore, S. D., Joshi, O. P., Ramesh, A. and Vyas, A. K., 2013. Productivity, sustainability and stability of soybean based cropping systems under different tillage systems. Soybean Research; 11 (1): 43-57.

Chander, N., Kumar, S., Ramesh and Rana, S.S., 2013 Nutrient removal by weeds and crops as affected by herbicide combinations in soybean-wheat cropping system. Indian Journal of Weed Science 45 (2): 99-105.

Chitale, Shrikant, Sarawgi, S.K., Tiwari, Alok and Urkurkar, J.S., 2011. Assessment of productivity and profitability of different rice (Oryza sativa) based cropping systems in Chhattisgarh plains. Indian Journal of Agronomy, 56 (4): 305-310.

Gallani, R., Sharma, S. K., Sirothia, P. and Joshi, O. P., 2013. Feasibility of organic farming system under soybean-wheat cropping sequence in Malwa region of Western Madhya Pradesh. Soybean Research; 11 (2): 62-69.

Gurjar, N., 2017. Performance of need based intensive cropping systems under irrigated condition in Rewa region of Madhya Pradesh. M.Sc. Agriculture thesis submitted to the JNKVV, Jabalpur.

Jadhav, A.S., 2014. Production potential of soybean-wheat cropping system 
through weed management. Indian Journal of Weed Science 46 (2): 190191.

Jugnahake M, Prajapat, R., Maurya, B.M. and Kurmvanshi, S.M., 2018. Identification of Cropping System Module for Irrigated Farming System of Rewa Region Int.J.Curr.Microbiol.App.Sci 7 (10): 687-694.

Karunakaran, V. and Behera, U.K. 2015. Tillage and residue management practices on yield, profitability, energy and water use efficiency in soybeanwheat cropping system. Experimental Agriculture doi: 10.1017/S0014479715000277.

Narkhede,W.N., Deshmukh, M.S., Bhale, V.M., Gill, M.S., Gadade, G.D. and More, S.S., 2011. Diversification of cropping systems under assured irrigation conditions in central plateau zone of Maharashtra. Indian Journal of Agronomy, 56(2): 104-108.

Narolia, R S, Meena, D S, Meena, H.P. Singh, P. and Nagar, B.L., 2018 Productivity, Profitability and Sustainability of Soybean (Glycine max)-wheat (Triticum aestivum) Cropping System as Influenced by Improved Water Management Technology in South Eastern Rajasthan. Soybean Research 16 (1 \& 2): 25-33.

Pradhan, A., Thakur, A., and Mukherjee, S.C., 2014 Weed dynamics and system productivity under rice-based cropping system. Indian Journal of Weed Science 46 (3): 224-228.

Prajapat, K., Vyas, A.K. and Dhar Shiva, 2014. Productivity, profitability and land use efficiency of soybean (Glycine max)-based cropping systems under different nutrient management practices. Indian Journal of Agronomy; 59 (2): 229-234.

Punia,S.S., Singh, S., Yadav, A., Yadav, D.B., and Malik, R.K., 2016. Long-term impact of crop establishment methods on weed dynamics, water use and productivity in rice-wheat cropping system. Indian Journal of Weed Science 48 (2): 158-163.

Ramesh, P., Panwar N. R. and Singh A. B., 2010. Crop productivity, soil fertility and economics of soybean (Glycine max), chickpea (Cicer arietinum) and blond psyllium (Plantago ovata) under organic nutrient management practices. Indian Journal of Agricultural Sciences, 80 (11): 965-969.

Ramesh, P., Panwar, N.R., Singh, A.B. and Ramana S., 2009. Production potential, nutrient uptake, soil fertility and economics of soybean (Glycine max)based cropping systems under organic, chemical and integrated nutrient management practices Indian Journal of Agronomy, 54 (3): 278- 283.

Sethi, I.B., Singh, R., Singh, V.K., Chaturvedi, S., Singh, A. P., Prabhakar, D. and Singh, J. P., 2018. Impact of crop intensification and establishment techniques on weed dynamics under different cropping systems. Indian Journal of Weed Science 50 (3): 262268.

Shrikant,C., Tiwari A., Bhoi S., Savu, R. M., Tomar, H. S. and Urkurkar, J.S., 2013. Performance of soybean (Glycine max)-based cropping sequences under organic, inorganic and integrated nutrient supply systems in a Vertisols. Indian Journal of Agronomy; 58 (2): 163-167.

Singh, D., Singh, H. and Ram, S., 2002. Impact of sowing dates, plant densities and farm yard manure on productivity of soybean (Glycine max). Extended Summaries Vol 1: 2nd International Agronomy Congress, Nov. 26- 30, New Delhi pp. 446-47.

Singh, D.K., Singh, R., Singh, G.D., Singh, 
A.P., Chaturvedi, S., Singh, J.P., Rathi, A. and Singh, M., 2017 Diversification of rice (Oryza sativa)-wheat (Triticum aestivum) system and its influence on productivity, profitability and energetic under on-farm situation. Indian Journal of Agronomy. 62 (3): 255-259.

Singh, A. K. and Kushwaha, H.S., 2018. Assessment of Soybean (Glycine max Merill L.) based cropping systems through Organic and Inorganic inputs in Bundelkhand Region. J Krishi Vigyan 6 (2): 7-12.
Soni, M., Upadhyay, V.B. and Singh, P., 2012. Weed dynamics and production efficiency of rice-based cropping system. Indian Journal of Weed Science 44(1): 21-25.

Walia, S.S., Gill, M.S., Bhushan, Bharat, Phuteja, R.R. and Aulakh, C.S., 2011. Alternate cropping system to rice (Oryza sativa)wheat (Triticum aestivum) for Punjab, Indian Journal of Agronomy, 56 (1): 20-27.

\section{How to cite this article:}

Gathiye, G. S. and Kushwaha, H. S. 2019. Effect of Soybean [Glycine max (L.) Merrill] based Cropping Systems on Weed Dynamics and Productivity of Soybean and Subsequent Crops of the System. Int.J.Curr.Microbiol.App.Sci. 8(10): 1626-1643.

doi: https://doi.org/10.20546/ijcmas.2019.810.190 